\title{
Epidermal cGVHD Score 3
}

National Cancer Institute

\section{Source}

National Cancer Institute. Epidermal CGVHD Score 3. NCI Thesaurus. Code C158594.

A score of 3 describing moderate disease with confluent erythema and scale and/or papules covering $>50 \%$ of target area. 